\title{
Effects of Surface Texture on Soft-Materials for Medical Applications
}

\author{
Kosuke Yasaka $^{1)^{*}}$, Yoshihiko Koseki ${ }^{2)}$, Kiyoshi Yoshinaka ${ }^{2)^{*}}$ and Koji Miyake ${ }^{2)}$ \\ ${ }^{1)}$ Togo Medikit Co., Ltd., \\ 1714-6 Kamekawa, Hichiya, Hyuga, Japan \\ ${ }^{2)}$ National Institute of Advanced Industrial Science and Technology (AIST) \\ 1-2-1 Namiki, Tsukuba, Ibaraki 305-8564, Japan \\ "Corresponding author: kousuke.yasaka@togomedikit.co.jp, k.yoshinaka@aist.go.jp \\ ( Manuscript received 01 September 2015; accepted 07 February 2016; published 30 April 2016 ) \\ ( Presented at the International Tribology Conference Tokyo 2015, 16-20 September, 2015 )
}

\begin{abstract}
We have studied the effects of a textured structures on soft-materials, mainly to reduce friction between body tissues and plastic components used in medical devices. For this purpose, we investigated changes in friction between the structure of grooves on plastic and rubber, which was considered as body tissue. Silicone oil was used for the purpose of lubrication, and the changes in friction were observed from the point of view of aspect ratio. The results indicated that a grooved surface exhibited lower friction compared to a non-textured surface. Furthermore, directional sliding perpendicular to the grooves was more effective in reducing friction than that parallel to the grooves. In addition, the results indicated that the kinetic friction was changed by pitch and depth even if the aspect ratio was similar in a direction perpendicular to the straight grooves. Furthermore, the results indicated that a higher aspect ratio resulted in a lower kinetic friction when the pitch was fixed and the depth was changed in a direction perpendicular to the straight grooves. In this study, the frictional materials used were soft-materials that simulated the softness of the human body and medical devices. Thus, from the results mentioned above, we can conclude that textured structures can reduce friction for soft materials.
\end{abstract}

Keywords: friction reduction, surface texture, lubrication, soft-materials, medical applications

\section{Introduction}

In order to improve the Quality of Life of patients, reducing the friction between medical devices and body tissues is urgently required in the medical field. For example, in the case of hemodialysis, patients undergoing dialysis are dialyzed approximately three times a week in Japan. Two indwelling needles are inserted into the patient's arm for performing dialysis. One of the needles sends blood from the patient's body to the dialyzer. The other needle sends filtered blood from the dialyzer to the patient's body. Thus, patients undergoing dialysis are frequently punctured with indwelling needles and they feel constant pains. In contrast to indwelling needles used for drip infusion, the friction generated on inserting an indwelling needle into a patient's arm for dialysis causes significant pain because the diameter of the needle is large. For effective dialysis, the needles have to ensure a sufficient quantity of blood flow. Thus, the diameter of the needle cannot be minimized to reduce the pain felt by the patient. Thus, a different solution is required that can reduce friction. Furthermore, in the case of endovascular treatment by using a catheter, the friction between the catheter and the inner wall of a blood vessel, and the friction between the inner part of the catheter and medical devices, such as guide wire, decreases the operability of medical devices. For example, if there is friction, the medical device cannot reach the affected area, and, in the worst-case scenario, the inner wall of a blood vessel may be damaged.

To solve these problems, lubricants, such as silicone oil, have been used conventionally for indwelling needles and other medical devices. Moreover, chemically modifying the surface of a medical device, such as hydrophilicity, has been used conventionally for catheters and guide wires. Furthermore, many researchers have carried out basic research on reducing friction for medical device applications. For example, Ikeuchi et al. reported that poly $(\mathrm{N}, \mathrm{N}$, dimethylacrylamide) (DMAA) grafted on the surface of a urethral catheter reduced friction in water because of its hydrophilic nature [1]. Sunita et al. reported that poly (MPC-co-BMA) coated on the surface of polyurethane reduced friction in water more than non-coated polyurethane because of hydration [2]. Yoshinaka et al. reported that low frequency oscillation reduced the 
surface friction of a medical instrument by changing contact condition [3]. To achieve a better friction reduction, an innovative solution is required.

In non-medical fields, such as the automobile industry and electronics, lubricated textured structures has been known as an innovative method for friction reduction, as compared to conventional lubrication and coating. The texture consists of shallow dimples or grooves processed in regular or irregular patterns on the friction surface $[2,4]$. These textures reduce real contact areas, maintain fluid film lubrication, prevent oil starvation, and generate hydrodynamic pressure [2,5-7]. Textured structures under lubrication have been actively investigated for friction reduction in engine cylinders and rotating shafts in hard disk drives (HDD) [8]. For example, Ryk et al. reported that the friction of piston rings whose surfaces were treated by partial laser surface texturing to create micro dimples was approximately $25 \%$ lower compared to non-textured piston [9]. Suh et al. reported that a micro-groove crosshatch pattern on the surface of steel could control friction under lubrication [10]. Ashwin et al. reported that the friction of textured stainless steel was approximately $80 \%$ lower compared to non-textured stainless steel [11].

Even though there have been various studies on geometries, such as grooves and dimples, the aspect ratio of depth to pitch is known as the major factor that influences a textured structure [6,10,12-13]. For example, Ronen et al. reported that the optimal aspect ratio was 0.12-0.14 when the surface of a piston ring was treated with laser to create dimples [14]. Wang et al. reported that an aspect ratio of 0.015 lead to the maximum load capacity of an oil film when SiC-textured surface was manufactured [15]. Costa et al. reported that an aspect ratio of 0.07 lead to the maximum film thickness [16].

The above-mentioned results were for hard materials such as metals. To apply textured structure to medical devices, the effects of a textured structure on soft-materials should be discussed because the human body is soft and the materials used in medical devices that are inserted into the body consists of soft plastic components.

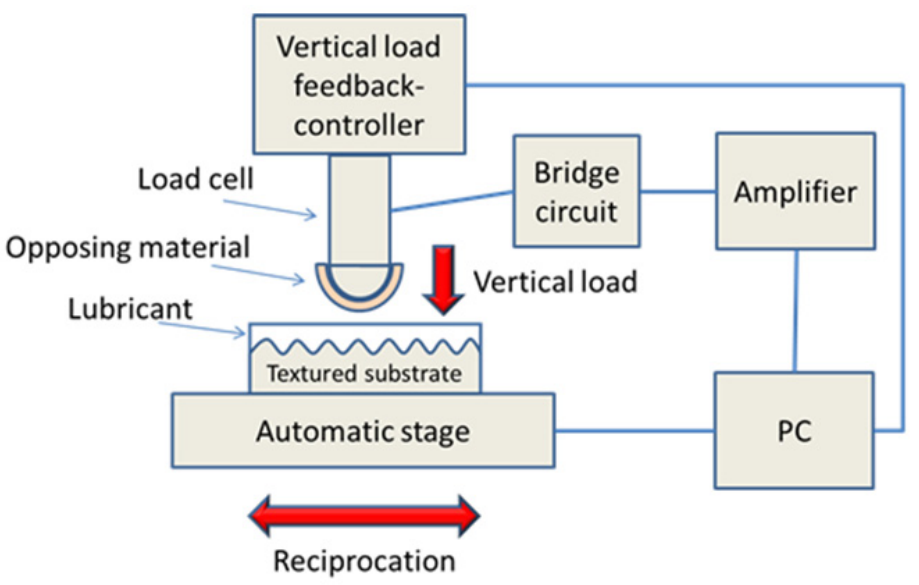

The effect of textured structure on soft-materials has been studied previously. For example, the textured structure of automobile tires has been studied to improve grip performance. Jian et al. investigated tire tread block friction characteristics and reported that different patterns of tread blocks have a certain influence on friction coefficient [17]. Hofstetter et al. studied a simulation procedure for sliding tread blocks under thermal effects and abrasion to develop a tire design [18]. Thus, the effect of textured structure on soft materials has been studied mainly to increase the friction of an automobile tire. On the other hand, we studied the effect of textured structure to reduce friction for medical devices and the tribology between soft-materials.

In this study, we studied the change in friction based on the aspect ratio obtained from the results of hard materials. First, we determined the frictional force between a plastic plate with processed straight grooves having an aspect ratio of 0.1 and silicone rubber that was used to simulate human tissue under lubricants. Second, we determined the frictional force between the plastic plate with processed straight grooves, whose aspect ratio was changed from 0.05 to 0.2 , and silicone rubber under the same conditions.

\section{Experimental methods}

We performed two types of experiments. The first experiment was performed using a plastic plate with processed straight grooves having an aspect ratio of 0.1 with various pitches and depths, as textured structures with an aspect ratio $=0.1$ has been commonly used [14-16]. The second experiment studied the effects of changing the aspect ratio from 0.05 to 0.2 .

In the experiments, to measure the effects of textured structure, we performed the experiments by using silicone rubber. Silicone rubber was repeatedly slid over a textured surface lubricated with silicone oil (as shown in Fig. 1). Two frictional directions were obtained. One direction was perpendicular to the straight grooves, and the second was parallel to the straight grooves (as shown in Fig. 2). The textured structure had straight grooves.

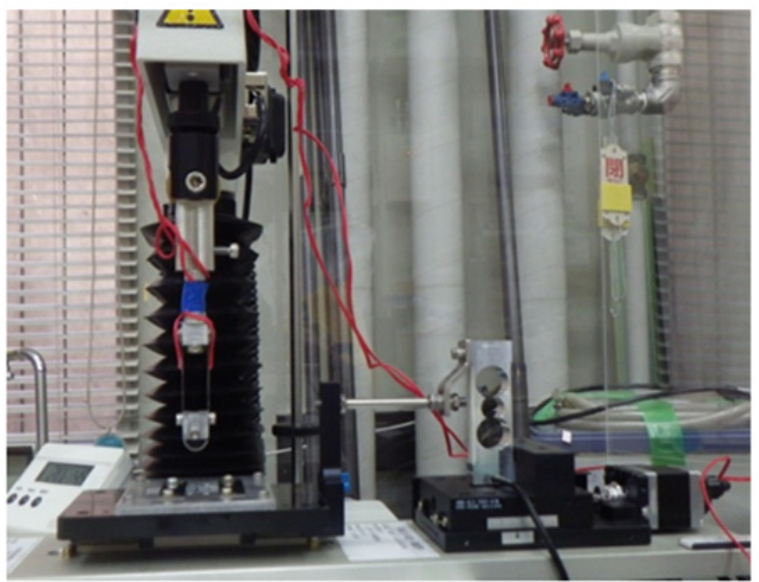

Fig. 1 Measurement system 


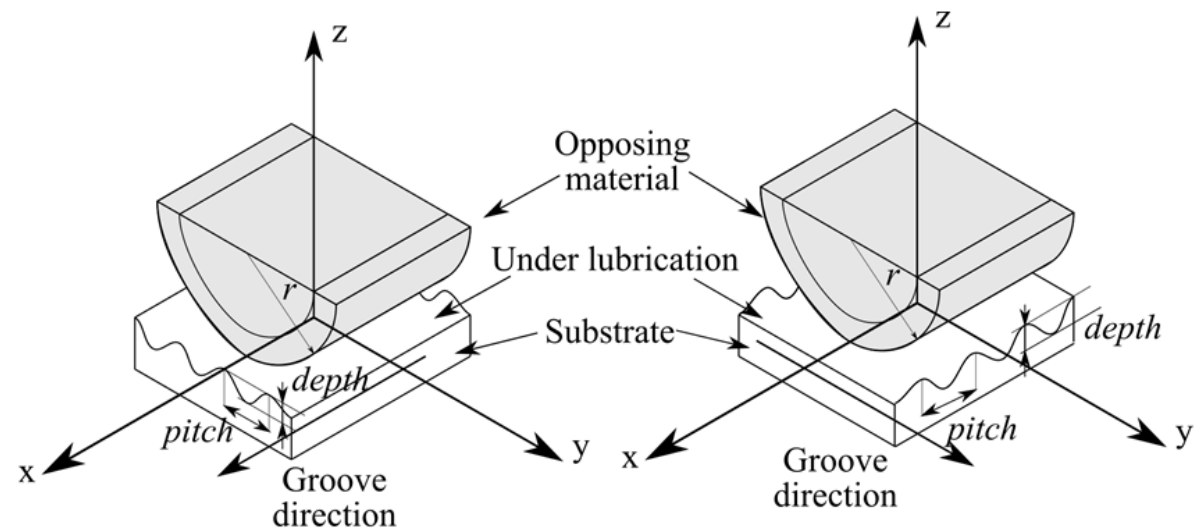

Fig. 2 Friction direction: a) perpendicular to the straight grooves, b) parallel to the straight grooves

The specifications of the textured structure are shown in Tables $(1,2)$. The pitch and depth of the straight grooves ranged between $25-100 \mu \mathrm{m}$ and 2.5-20 $\mu \mathrm{m}$ respectively. Straight grooves with aspect ratios of $0.13,0.10$, and 0.08 were assumed as grooves with aspect ratio of 0.1 , as the depth of grooves was not precisely controllable in our process. The surface of a plastic plate (Polypropylene, Hazaiya Co., Ltd., $15 \mathrm{~mm} \times 15 \mathrm{~mm} \times 1 \mathrm{~mm})$ was processed to create a textured structure by pressing a metal mold (aluminum alloy) having a textured structure. Before processing the plastic plate was washed with isopropyl alcohol (Wako Pure Chemical Industries, Ltd.). The textured structure was processed on the metal mold by using a shaper. The metal mold was pressed into the plastic plate by using a vice and a hot plate (EC Hotplate EC-1200N, AS ONE Corporation) under heated conditions for the purpose of texturing. The heat was set at $25-120^{\circ} \mathrm{C}$ depending on each textured structure. The textured structure on the surface of the plastic plate was confirmed using a confocal laser microscope (KEYENCE CORPORATION, VK-9510), as shown in Fig. 3, the textured samples were tested and compared

Table 1 Specifications of textured structure (Aspect ratio around 0.1)

\begin{tabular}{|c|c|c|c|}
\hline Sample & $\mathrm{P}($ Pitch $\mu \mathrm{m})$ & $\mathrm{D}($ Depth $\mu \mathrm{m})$ & Aspect Ratio(D/P) \\
\hline P100-D13-AR0.13 & 100 & 13 & 0.13 \\
\hline P50-D4-AR0.08 & 50 & 4 & 0.08 \\
\hline P25-D2.5-AR0.10 & 25 & 2.5 & 0.10 \\
\hline Non textured sample & - & - & - \\
\hline
\end{tabular}

Table 2 Specifications of textured structure (pitch was fixed $100 \mu \mathrm{m}$ )

\begin{tabular}{|c|c|c|c|}
\hline Sample & $\mathrm{P}($ Pitch $\mu \mathrm{m})$ & $\mathrm{D}($ Depth $\mu \mathrm{m})$ & Aspect Ratio(D/P) \\
\hline P100-D20-AR0.20 & 100 & 20 & 0.20 \\
\hline P100-D13-AR0.13 & 100 & 13 & 0.13 \\
\hline P100-D4.8-AR0.05 & 100 & 4.8 & 0.05 \\
\hline
\end{tabular}
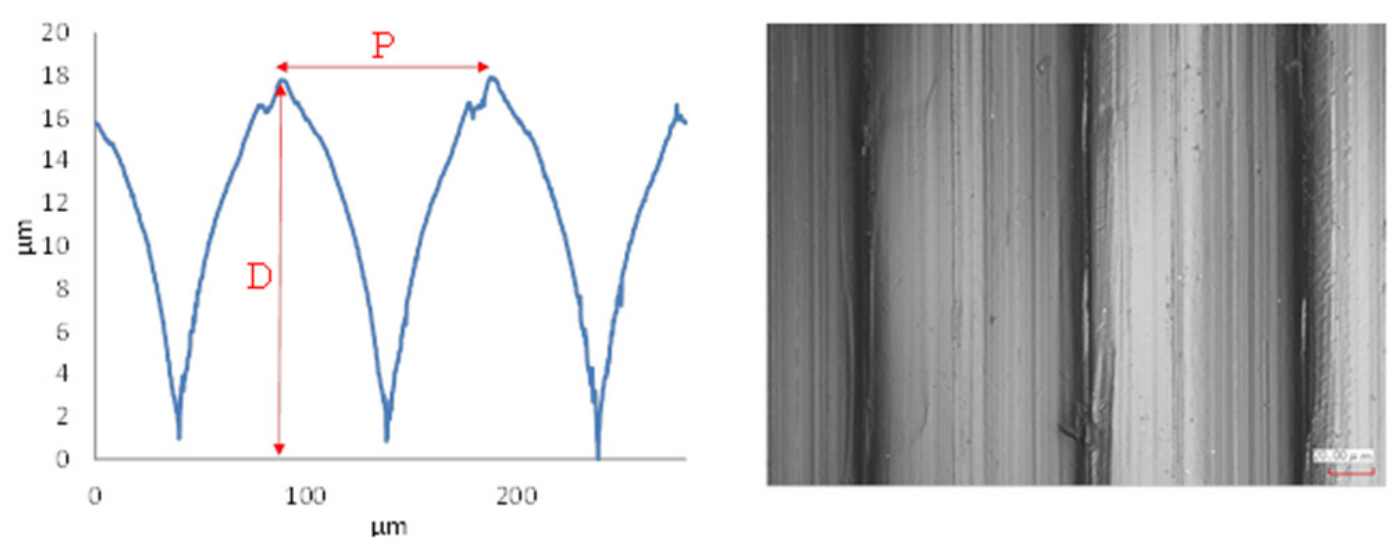

Fig. 3 Textured structure (P: pitch; D: depth) 


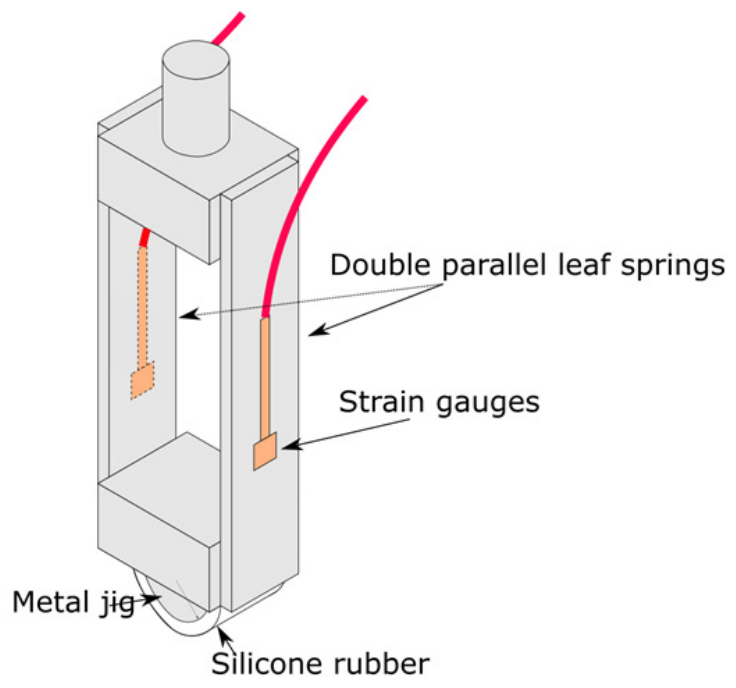

Fig. 4 Friction measurement system

with non-textured samples. The opposing material was silicone rubber (Kyowa Industries Inc, $5 \mathrm{~mm} \times 10 \mathrm{~mm}$, Shore hardness 10 degrees), which was used to simulate the softness of the human body. Silicone rubber was adhered to a circular metal jig ( $\varphi 10$, half cylinder, S45C, MISUMI Corporation) in order to press on set load.

The frictional force between the textured surface and silicone rubber was measured using strain gauges attached to double parallel leaf springs (Fig. 4). The circular metal jig adhered to silicone rubber was fixed on the double parallel leaf springs. Vertical load was set at a constant value and controlled using a feedback controller (Texture Analyzer ${ }^{\circ}$ Texture Technologies Corp and by stable micro systems, Ltd). The range of vertical load was between 0.2-2.0 N. The samples were reciprocated using an automatic stage (SIGMAKOKI Co., Ltd). The length of the stroke was $5 \mathrm{~mm}$. The sliding speed was set at $10 \mathrm{~mm} / \mathrm{s}$, and the number of friction cycles was 40 . The lubricant used was silicone oil (Dow corning, MDX4-4159 50\% medical grade dispersion). The viscosity of the silicone oil was $170 \mathrm{cst}\left(170 \mathrm{~mm}^{2} / \mathrm{s}\right)$, and $10 \mathrm{ml}$ of the lubricant was applied on the textured surface in the form of drops.

\section{Results}

3.1. Validation of results for constant aspect ratio (The first experiment)

We studied the samples that had an aspect ratio of 0.1 . The samples had different depths and pitches. The kinetic friction was determined by the system and shown in Fig. 1. Figures 5,6 show the relationship between the kinetic friction and vertical load. Figure 5 shows the results for the friction direction perpendicular to the straight grooves. Figure 6 shows the results for the friction direction parallel to the straight grooves.

\subsubsection{Friction along direction perpendicular to straight grooves}

As shown in Fig. 5, a higher vertical load resulted in a higher kinetic friction. The kinetic friction decreased in the following order: Non-textured surface > P25-D2.5-AR0.10 > P50-D4-AR0.08 > P100-D13-AR0.13 (As shown in Table 1. P: pitch, D: depth, AR: aspect ratio, and the rest are omitted). Every sample did not exhibit significant differences in kinetic friction until a vertical load of $0.8 \mathrm{~N}$ was applied. However, the samples were confirmed to have significant differences in kinetic friction when the vertical load exceeded $1.0 \mathrm{~N}$; kinetic frictions of the non-textured sample, P25-D2.5-AR0.10, and P50-D4-AR0.08 increased significantly. Finally, when the vertical load was $2.0 \mathrm{~N}$, which was the maximum value, kinetic frictions of the non-textured sample, P25-D2.5-AR0.10, and P50-D4-AR0.08 were $0.82 \mathrm{~N}, 0.56 \mathrm{~N}$, and $0.36 \mathrm{~N}$ respectively. P25-D2.5-AR0.10 exhibited approximately $32 \%$ lower kinetic friction compared to that of the non-textured sample. P50-D4-AR0.08 exhibited approximately $56 \%$ lower kinetic friction compared to that of the non-textured sample. On the contrary, the kinetic friction of P100-D13-AR0.13 increased significantly when the vertical load exceeded $1.8 \mathrm{~N}$. Finally, when the vertical load was $2.0 \mathrm{~N}$, the kinetic friction increased to $0.15 \mathrm{~N}$. P100-D13-AR0.13 exhibited approximately $82 \%$ lower kinetic friction

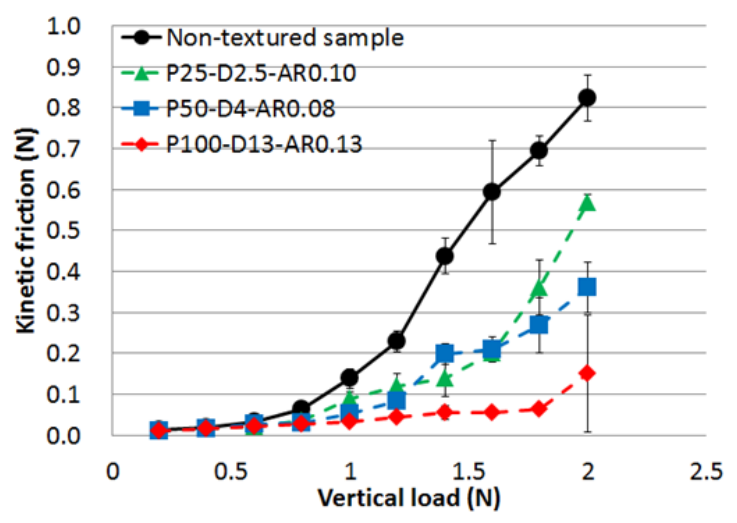

Fig. 5 Kinetic friction (Direction perpendicular to the straight grooves)

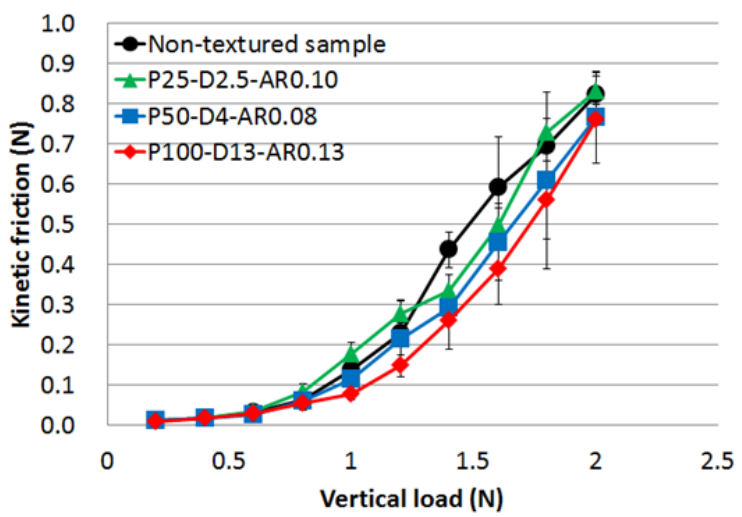

Fig. 6 Kinetic friction (Direction parallel to the straight grooves) 
compared to that of the non-textured sample.

As a result, kinetic frictions of P25-D2.5-AR0.10, P50-D4-AR0.08, and P100-D13-AR0.13 decreased compared to the non-textured sample. The relationship trend between the kinetic friction and vertical load for P25-D2.5-AR0.10 was similar to that of P50-D4-AR0.08. P100-D13-AR0.13 had a low kinetic friction under a high vertical load. Therefore, P100-D13-AR0.13, which has a pitch of $100 \mu \mathrm{m}$ and a depth of $13 \mu \mathrm{m}$, had the lowest friction in this study. These results show that kinetic friction depends on the size of grooves even if the aspect ratio is the same.

3.1.2 Friction along direction parallel to straight grooves

As shown in Fig. 6, a higher vertical load resulted in a higher kinetic friction, which is true in case the friction direction is perpendicular to the straight grooves. The kinetic friction decreased in the following order: Non-textured sample $>$ P25-D2.5-AR0.10 > P50-D4-AR0.08 > P100-D13-AR0.13. Every sample did not exhibit significant differences in kinetic friction until a vertical load of $0.8 \mathrm{~N}$ was applied. However, all the samples were confirmed to have slight differences in kinetic friction when the vertical load exceeded $1.0 \mathrm{~N}$. Finally, when the vertical load was $2.0 \mathrm{~N}$, kinetic frictions of the non-textured sample, P25-D2.5-AR0.10, P50-D4-AR0.08, and P100-D13-AR0.13 were $0.83 \mathrm{~N}$, $0.83 \mathrm{~N}, 0.76 \mathrm{~N}$, and $0.76 \mathrm{~N}$, respectively. Compared to the non-textured sample, kinetic frictions of the texture samples decreased. Nonetheless, a significant difference could not be confirmed between the samples.

As a result, kinetic friction in a direction parallel to the straight grooves did not decrease as significantly as the kinetic friction in a direction perpendicular to the straight grooves. In addition, it is assumed that kinetic friction decreased slightly in a direction parallel to the straight grooves compared to the non-textured structure mainly because the contact area between the textured structure and the opposing material decreased.

3.1.3 Comparison between direction perpendicular to straight grooves and direction parallel to straight grooves

As described in section 3.1.1, kinetic frictions of all textured samples were significantly lower than non-textured samples in a direction perpendicular to the straight grooves. In section 3.1.2, the kinetic frictions of all textured samples were slightly lower than the non-textured sample in a direction parallel to the straight grooves. Comparing the kinetic friction of P100-D13-AR0.13 in a direction perpendicular to the straight grooves to that in a direction parallel to the straight grooves under a vertical load of $2.0 \mathrm{~N}$, it can be observed that kinetic friction in a direction perpendicular to the straight grooves was $0.15 \mathrm{~N}$, and that in a direction parallel to the straight grooves was $0.76 \mathrm{~N}$. Thus, kinetic friction in a direction perpendicular to the straight grooves exhibited approximately $80 \%$ lower compared to that in a direction

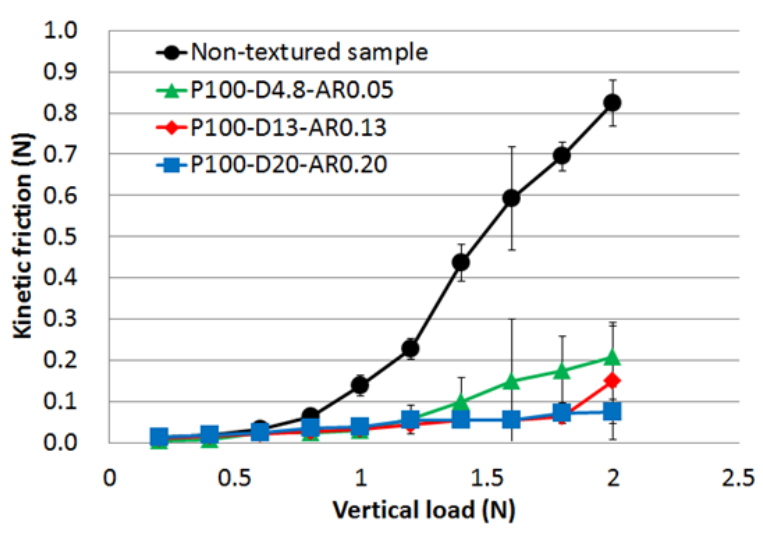

Fig. 7 Kinetic friction (different aspect ratios)

parallel to the straight grooves. Therefore, kinetic friction in a direction perpendicular to the straight grooves decreased more in comparison to that in a direction parallel to the straight grooves.

3.2. Comparison of different aspect ratios (constant pitch of $100 \mu \mathrm{m})$ (the second experiment)

As described in Section 3.1.1, kinetic friction at an aspect ratio of $0.13(100 \mu \mathrm{m}$ pitch, $13 \mu \mathrm{m}$ depth) was lower than other samples in a direction perpendicular to the straight grooves. Therefore, a pitch of $100 \mu \mathrm{m}$ had the potential to decrease kinetic friction. Thus, we tested samples with different aspect ratios (the pitch was fixed at $100 \mu \mathrm{m}$, the depth was 20 and $4.8 \mu \mathrm{m}$ ) (Shown in Table 2).

Figure 7 shows the relationship between the kinetic friction and vertical load (in a direction perpendicular to the straight grooves). The kinetic friction decreased in the following order: non-textured sample > P100-D4.8-AR0.05 > P100-D13-AR0.13 > P100-D20-AR0.20. As a result, samples having a bigger aspect ratio had a lower kinetic friction. The kinetic friction of P100D4.8-AR0.05 started to increase when the vertical load exceeded $1.2 \mathrm{~N}$ and increased to $0.20 \mathrm{~N}$. The kinetic friction of P100-D13-AR0.13 started to increase when the vertical load exceeded $1.8 \mathrm{~N}$ and increased to $0.15 \mathrm{~N}$. The kinetic friction of P100-D20-AR0.20, which changed slightly from 0.01 to $0.08 \mathrm{~N}$, did not increase while the vertical load was in the range of 0.2-2.0 N. Hence, it can be observed that a higher aspect ratio resulted in a lower kinetic friction.

\section{Discussion}

We obtained mainly three results, which are described as follows;

- The kinetic friction in a direction perpendicular to the straight grooves decreased more in comparison to that in a direction parallel to the straight grooves.

- The kinetic friction changed for different values of pitch and depth even if the aspect ratio was the same in a direction perpendicular to the straight grooves. 

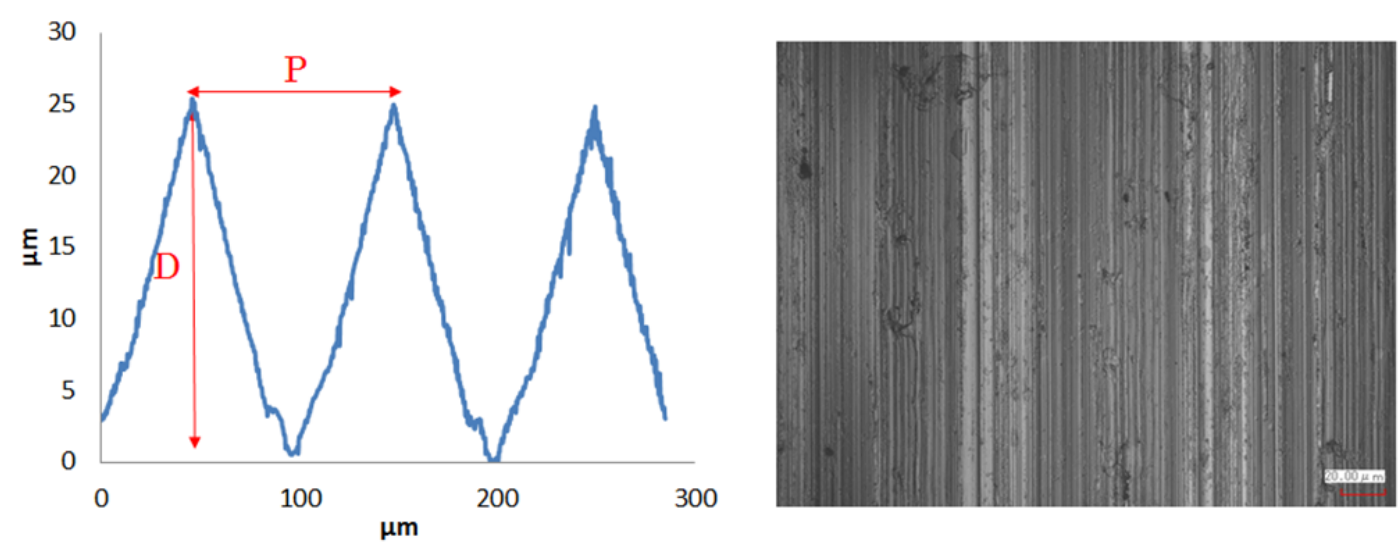

Fig. 8 Textured metal

- When the pitch was fixed and the depth was changed, a higher aspect ratio resulted in a lower kinetic friction in a direction perpendicular to the straight grooves.

From the results, we observed that the kinetic friction in a direction perpendicular to the straight grooves was significantly lower than that in a direction parallel to the straight grooves. This trend had been observed in previous studies in machinery fields. For example, in a strip-drawing process, friction occurs between a metal strip and the two dies. Costa et al. studied the effects of a textured structure to reduce friction in a strip-drawing process. They reported that the frictional force of grooves, which were oriented in a perpendicular direction to the drawing direction, was considerably reduced compared to grooves oriented in a parallel direction [19]. In a fundamental study, Sihuan et $a l$. reported that grooves perpendicular to the sliding direction had a better effect on friction reduction than that in a parallel orientation under a low contact pressure [20]. They studied the effect of a lubricated textured structure on friction for hard materials. In this study, we studied the effect of a lubricated textured structure on friction for soft-materials that are used in medical practice. Therefore, we studied the effect of textured structures for hard materials using the same conditions to determine if the effect of textured structure on reducing friction is the same for both hard and soft materials. We determined the frictional force between a metal (aluminum alloy), which was processed with straight grooves having a pitch of $100 \mu \mathrm{m}$ and a depth of $25 \mu \mathrm{m}$ (As shown in Fig. 8, similar to P100-D20-AR0.20), and another opposing metal (S45C, MISUMI Corporation) under lubricated conditions (under the same conditions used in the first and second experiments except that textured substrate and opposing material were different). Moreover, we determined the frictional force between a non-textured metal (aluminum alloy) and the textured metal (S45C).

The results are shown in Fig. 9. A significant difference could not be confirmed between the non-textured metal and textured metal. Thus, textured structures could not reduce friction in metals under

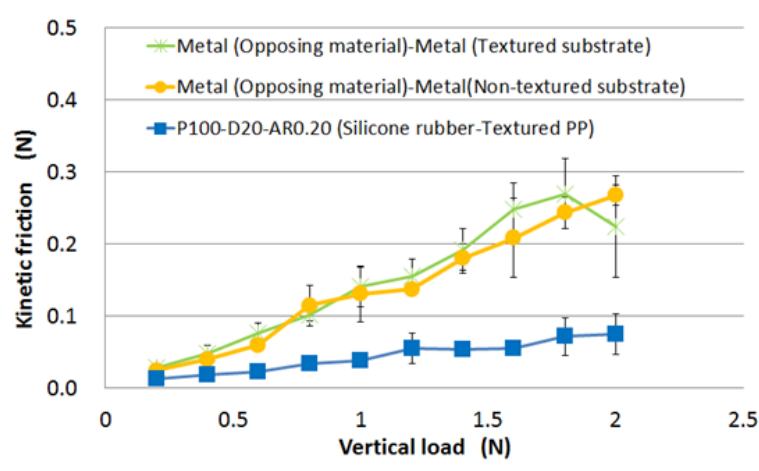

Fig. 9 Kinetic friction (soft materials vs hard materials)

these conditions (the shape of the textured structure, viscosity of the lubricant, and sliding speed). It was also confirmed that the kinetic friction between hard materials (the textured metal and another opposing metal) was higher than that between soft-materials (the textured polypropylene and the opposing silicone rubber). Finally, under a vertical load of $2.0 \mathrm{~N}$, the kinetic friction between soft-materials was $0.08 \mathrm{~N}$ and that between hard materials was $0.22 \mathrm{~N}$. The kinetic friction between soft-materials was approximately $66 \%$ lower compared to that between hard materials. Therefore, we assume that textured structures only reduce friction between soft-materials when the shape of the textured structure, viscosity of the lubricant, and sliding speed are considered. Therefore, we believe that these effects that reduce the friction between soft materials are specific only to soft materials.

In this study, we assume that the opposing material contacts a rib of the textured substrate, as shown in Fig. 10. As the opposing material is softer than the textured substrate, when a vertical load is applied, the opposing material deforms and presses into the grooves. The lubricant is held in the grooves. We believe that the following phenomena occurred when the opposing material slid on the textured surface under the conditions mentioned above:

1) The effect of hydrodynamic pressure

2) Scraping out lubricant by deformation of the 


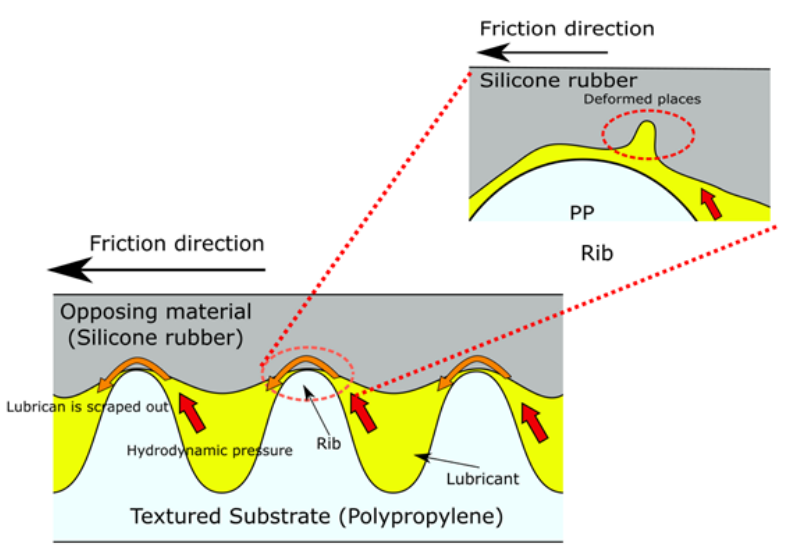

Fig. 10 Hypothesis

opposing material

3) The effect of soft EHL

4) Amount of lubricant in grooves

5) The effect of viscoelastic dissipation

\subsection{The effect of hydrodynamic pressure}

Hydrodynamic pressure is regarded as the most important effect of textured surfaces [20-23]. In this study, in the case where the sliding direction was perpendicular to the straight grooves, the cross-section of the grooves was an inverted triangle (As shown in Fig. 3). Therefore, the grooves were wedge-shaped in the direction of friction. The wedge shape is one of the most important factors to generate hydrodynamic pressure [8]. Nanbu et al. studied the effect of hydrodynamic action by using model-based virtual texturing and numerical simulation, and they observed that different hydrodynamic effects were produced depending on the texture of the bottom surface. They reported that the oil film forming performance was high when the bottom surface had a wedge-shaped texture. [8,24]. Thus, we believe that the hydrodynamic pressure is generated in the grooves in a direction perpendicular to the straight grooves. The behavior of kinetic friction depended on the shape of grooves (pitch and depth). We believe that a bigger cross-sectional shape results in a higher hydrodynamic pressure and this reduces friction. More studies describing the relationship between the shape of the grooves and the hydrodynamic pressure are required in the future.

In the case where the frictional direction is parallel to the straight grooves, the hydrodynamic pressure is not generated in the grooves. This is because the straight grooves are in the same direction as the frictional direction.

Thus, the kinetic friction in a direction perpendicular to the straight grooves was significantly lower than that in a direction parallel to the straight grooves, and there was no significant difference between non-textured samples and textured samples in a direction parallel to the straight grooves.
4.2. Scraping out lubricant by deformation of the opposing material

Both the opposing material and textured substrate were soft-materials. The Young's modulus of polypropylene used as the textured substrate was approximately $1.5 \times 10^{3} \mathrm{MPa}$. In contrast, the Young's modulus of silicone rubber used as opposite material was approximately 4.0 $\mathrm{MPa}$. Thus, silicone rubber as the opposing material was softer than polypropylene as the textured substrate. Therefore, the opposing material was deformed by the vertical load and pressed into the texture grooves when the vertical load was applied.

When the opposing material slides on the textured surface in a direction perpendicular to the straight grooves, the opposing material pressed into grooves scraps out the lubricant existing in a groove. Consequently, this phenomenon results in lubricating other grooves. Thus, the lubricant is constantly held in grooves (As shown in Fig. 10).

When the opposing material slides on the textured surface in a direction parallel to the straight grooves, the opposing material does not scrap out the lubricant existing in a groove because the straight grooves are in the same direction as the frictional direction. Thus, the lubricant is not provided to other grooves. Thus, oil starvation occurs easily compared to that in a direction perpendicular to the straight grooves. Therefore, as shown in Figs. 5,6, as well as described in section 4.1, the kinetic friction in a direction perpendicular to the straight grooves was significantly lower than that in a direction parallel to the straight grooves.

\subsection{The effect of soft EHL}

As described in sections 4.1 and 4.2, in case the sliding direction is perpendicular to the straight grooves, hydrodynamic pressure is generated in the grooves, and the opposing material scraps out lubricant existing in the textured grooves; therefore, lubricant is provided to other grooves. In addition, we believe that soft EHL generated at contact areas between the opposing material and the textured surface at the ribs had an effect on friction reduction [25]. Silicone rubber was softer than polypropylene. We believe that when the opposing material slides on the textured surface, the opposing material deforms at the contact areas because the hydrodynamic pressure is generated in the grooves. The hydrodynamic pressure increases as the area to flow lubricant narrows [8,24-25]. Thus, the hydrodynamic pressure increases most at the lubricant inlet of a rib. Thus, the opposing material deforms most at the lubricant inlet of the rib. We believe that when the opposing material deforms, the lubricant is held in the deformed place at a microscopic level; thus, the lubricant is held at the contact areas (As shown in Fig. 10). If this effect does not occur, the lubricant flows laterally as the lubricant cannot flow forward, and lubricant cannot be provided at the contact areas in the rib. Thus, oil starvation occurs. However, the kinetic friction 
decreased in this study. Therefore, we believe that the effect of soft EHL may also reduce friction. However, we did not study the above phenomenon experimentally. To discuss this mechanism in detail, we need to study the above effects by using visualization experiment in the future.

\subsection{Amount of lubricant in grooves}

As described in sections 3.1 and 3.2, in case the friction direction is perpendicular to the straight grooves, the kinetic friction changed for different values of pitch and depth even if the aspect ratio was the same, and a larger pitch resulted in a lower kinetic friction. We also observed that when the pitch was fixed and the depth was changed, a higher aspect ratio resulted in a lower kinetic friction. We think that these results are related to the amount of lubricant in the grooves. Thus, we tried to compare the amount of lubricant in grooves (As shown in Fig. 11 and Table 3). In the first experiment, compared to the oil groove cross-sectional area having a width of 280 $\mu \mathrm{m}$, the cross-sectional area of P100-D13-AR0.13 was $1083 \mu^{2}$ and that of P50-D4-AR0.08 was $363 \mu \mathrm{m}^{2}$ (third part of P100-D13-AR0.13). The area of P25-D2.5-AR0.10 was $311 \mu \mathrm{m}^{2}$ (third point fifth part of D100-D13-AR0.13). As shown in Fig. 5, when the vertical load was $2.0 \mathrm{~N}$, the kinetic frictions of P25-D2.5-AR0.10 (311 $\left.\mu^{2}{ }^{2}\right), \quad$ P50-D4-AR0.08 (363 $\left.\mu \mathrm{m}^{2}\right)$, and P100-D13-AR0.13 $\left(1083 \mu \mathrm{m}^{2}\right)$ were $0.56 \mathrm{~N}$, $0.36 \mathrm{~N}$, and $0.15 \mathrm{~N}$, respectively. The kinetic friction decreased with increasing oil groove area. This result was also confirmed in the second experiment. As shown in Table 4, the area of the oil groove increased with increasing the depth of the groove. Compared to the oil groove cross-sectional area having a width of $280 \mu \mathrm{m}$, the cross-sectional areas of P100-D20-AR0.20, P100-D13-AR0.13, P100-D4.8-AR0.05 were $1815 \mu \mathrm{m}^{2}$, $1083 \mu \mathrm{m}^{2}$, and $826 \mu \mathrm{m}^{2}$, respectively As shown in Fig.7, when the vertical load was $2.0 \mathrm{~N}$, the kinetic frictions of P100-D4.8-AR0.05 (826 $\mu \mathrm{m}^{2}$ ), P100-D13-AR0.13 (1083 $\left.\mu \mathrm{m}^{2}\right)$, and P100-D20-AR0.20 $\left(1815 \mu \mathrm{m}^{2}\right)$ were $0.20 \mathrm{~N}$, $0.15 \mathrm{~N}$, and $0.08 \mathrm{~N}$, respectively. The kinetic friction also decreased with increasing oil groove area. Thus, textured samples, which are processed with a large oil groove area, have a low kinetic friction in order to maintain fluid film lubrication. Oil starvation is generated easily in textured samples processed with a small oil groove area when the vertical load increases because the opposing material deforms and scraps out lubricant in the grooves based on the vertical load. As a result, it was observed that the kinetic friction varied based on the depth and pitch even if the aspect ratio was the same. Furthermore, when the pitch was fixed and the depth was changed, a higher aspect ratio resulted in a lower kinetic friction.

On the other hand, we believe that, except the amount of lubricant, the pitch and depth of the textured structure (shape of the textured structure), and the deformation volume of the opposing material are also related to the hydrodynamic pressure (section 4.1) Thus we think it is necessary to set an optimal pitch and depth

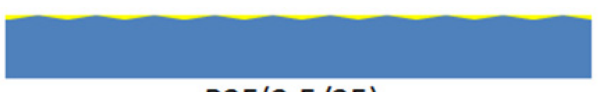

$\mathrm{P} 25(2.5 / 25)$
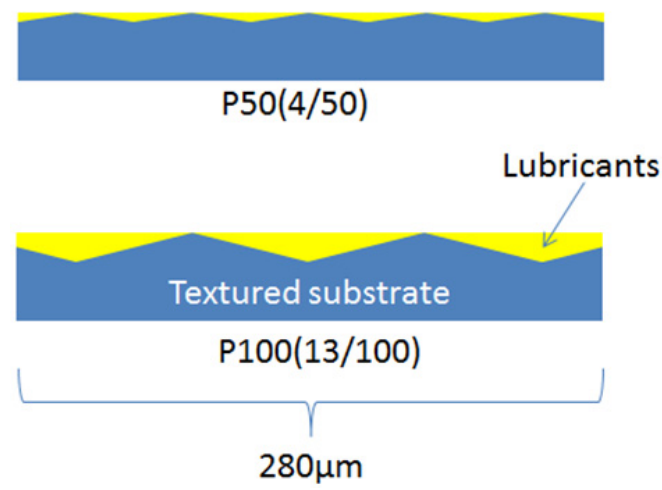

Fig. 11 Comparison of oil grooves

Table 3 Lubricant area comparison of lubricant's cross-section area for $280 \mu \mathrm{m}$ (Aspect ratio around 0.1)

\begin{tabular}{|c|c|c|c|c|}
\hline Sample & Aspect ratio & Depth $(\mu \mathrm{m})$ & Pitch $(\mu \mathrm{m})$ & $\begin{array}{c}\text { Lubricant Area } \\
(\text { Range } 280 \mu \mathrm{m}) \\
\left(\mu \mathrm{m}^{2}\right)\end{array}$ \\
\hline P100-D13-AR0.13 & 0.13 & 13 & 100 & 1083 \\
\hline P50-D4-AR0.08 & 0.08 & 4 & 50 & 363 \\
\hline P25-D2.5-AR0.10 & 0.10 & 2.5 & 25 & 311 \\
\hline
\end{tabular}

Table 4 Lubricant area comparison of lubricant's cross-section area for $280 \mu \mathrm{m}$ (pitch was fixed $100 \mu \mathrm{m}$ )

\begin{tabular}{|c|c|c|c|c|}
\hline Sample & Aspect ratio & Depth $(\mu \mathrm{m})$ & Pitch $(\mu \mathrm{m})$ & $\begin{array}{c}\text { Lubricant Area } \\
(\text { Range } 280 \mu \mathrm{m}) \\
\left(\mu \mathrm{m}^{2}\right)\end{array}$ \\
\hline P100-D20-AR0.20 & 0.20 & 20 & 100 & 1815 \\
\hline P100-D13-AR0.13 & 0.13 & 13 & 100 & 1083 \\
\hline P100-D4.8-AR0.05 & 0.05 & 4.8 & 100 & 826 \\
\hline
\end{tabular}


in view of the hydrodynamic pressure, amount of lubrication, and deformation volume of the opposing material. More detailed studies are required in the future.

\subsection{The effect of viscoelastic dissipation}

As the opposing material used in this study was rubber, viscoelastic dissipation should be discussed. Viscoelastic dissipation affects friction more effectively when the opposing materials directly contact the textured substrate without a lubricant. However, on the application of a lubricant, friction is reduced compared to that with viscoelastic dissipation. Thus, we believe that other effects (section 4.1-4.4) mainly affect friction compared to viscoelastic dissipation. However, we believe that the effect of viscoelastic dissipation is also important; therefore, we need to study it in more detail in the future.

We believe that the effects mentioned above interact in a complex manner and reduces the kinetic friction by acting simultaneously when a material is slid on another. Apart from these effects, there may be other effects also that may affect the kinetic friction of sliding materials. Thus, more studies are required to understand the effect of textured structures on reducing friction between soft materials.

In this study, we confirmed that the kinetic friction between silicone rubber that simulated the softness of the human body and polypropylene used for medical devices was reduced by processing a textured structure. Therefore, we believe that a textured structure has the potential to reduce friction caused between medical devices and body tissue in the future. In addition, this study contributes to a fundamental study of soft EHL.

\section{Conclusion}

Our findings are summarized as follows:

- The kinetic friction in a direction perpendicular to the straight grooves decreased more compared to that in a direction parallel to the straight grooves.

- The kinetic friction changed for different values of pitch and depth even if the aspect ratio was the same in a direction perpendicular to the straight grooves.

- When the pitch was fixed and the depth was changed, a higher aspect ratio resulted in a lower kinetic friction in a direction perpendicular to the straight grooves.

- The kinetic friction between soft-materials was lower compared to that between hard materials.

- We believe that these effects that reduce the friction between soft materials are specific only to soft materials.

\section{Acknowledgments}

We gratefully acknowledge to Dr. Y. Usui of AIST for his help in the fabrication of textured samples, and also
Dr. A. Korenaga of AIST for fruitful discussion about the EHL theory.

We would like to thank Editage (www.editage.jp) for English language editing.

\section{References}

[1] Ikeuchi, K., Takii, T., Norikane, H., Tomita, N., Ohsumi, T., Uyama, Y. and Ikada, Y., "Water Lubrication of Polyurethane Grafted with Dimethylacrylamide for Medical Use," Wear, 161, 1-2, 1993, 179-185.

[2] Ho, S. P, Nakabayashi, N., Iwasaki, Y., Boland, T. and LaBerge, M., "Frictional Properties of Poly (MPC-co-BMA) Phospholipid Polymer for Catheter Applications," Biomaterials, 24, 28, 2003, 5121-5129.

[3] Yoshinaka, K., Takashima, K., Okazaki, T., Ikeuchi, K., Washio, T. and Chinzei, K., "Experimental Study to Control the Insertion Resistance of Internal Medical Instrument Using Magnetic Field Oscillation," Tribology International, 40, 2, 2007, 339-344.

[4] Nakano, M., Korenaga, A., Korenaga, A., Miyake, K., Murakami, T., Ando, Y., Usami, H. and Sasaki, S., "Applying Micro-Texture to Cast Iron Surfaces to Reduce the Friction Coefficient under Lubricated Conditions," Tribology Letters, 28, 2, 2007, 131-137.

[5] Kovalchenko, A., Ajayi, O., Erdemir, A., Fenske, G. and Etsion, I., "The Effect of Laser Surface Texturing on Transitions in Lubrication Regimes During Unidirectional Sliding Contact," Tribology International, 38, 3, 2005, 219-225.

[6] Kovalchenko, A., Ajayi, O., Erdemir, A., Fenske, G. and Etsion, I., "The Effect of Laser Texturing of Steel Surfaces and Speed-Load Parameters on the Transition of Lubrication Regime from Boundary to Hydrodynamic," Tribology Transactions, 47, 2, 2004, 299-307.

[7] Andersson, P., Koskinen, J., Varjus, S., Gerbig, Y., Haefke, H., Georgiou, S., Zhmud, B. and Buss, W., "Microlubrication Effect by Laser-Textured Steel Surfaces," Wear, 262, 3-4, 2007, 369-379.

[8] Korenaga, A., "Shapes and Dimensions of Surface Textures and their Tribological Properties," Journal of Japanese Society of Tribologists, 55, 2, 2010, 101-106 (in Japanese).

[9] Ryk, G. and Etsion, I., "Testing Piston Rings with Partial Laser Surface Texturing for Friction Reduction," Wear, 261, 7-8, 2006, 792-796.

[10] Suh, M., Chae, Y., Kim, S., Hinoki, T. and Kohyama, A., "Effect of Geometrical Parameters in Micro-Grooved Crosshatch Pattern Under Lubricated Sliding Friction," Tribology International, 43, 8, 2010, 1508-1517.

[11] Ramesh, A., Akram, W., Mishara, S. P., Cannon, A. H., Polycarpou, A. A. and King, W. P., "Friction 
Characteristics of Microtextured Surfaces Under Mixed and Hydrodynamic Lubrication," Tribology International, 57, 2013, 170-176.

[12] Etsion, I., Kligerman, Y. and Halperin, G., "Analytical and Experimental Investigation of Laser-Textured Mechanical Seal Faces," Tribology Transactions, 42, 3, 1999, 511-516.

[13] Brizmer, V., Kligerman, Y. and Etsion, I., "A Laser Surface Textured Parallel Thrust Bearing," Tribology Transactions, 46, 3, 2003, 397-403.

[14] Ronen, A., Etsion, I. and Kligerman, Y., "Friction-Reducing Surface-Texturing in Reciprocating Automotive Components," Tribology Transactions, 44, 3, 2001, 359-366.

[15] Wang, X., Kato, K. and Adachi, K., "The Lubrication Effect of Micro-Pits on Parallel Sliding Faces of SiC in Water," Tribology Transactions, 45, 3, 2002, 294-301.

[16] Costa, H. L. and Hutchings, I. M., "Hydrodynamic Lubrication of Textured Steel Surfaces Under Reciprocating Sliding Conditions," Tribology International, 40, 8, 2007, 1227-1238.

[17] Wu, J., Wang, Y. S., Su, B. L. and Liu, Q., "Experimental and Numerical Studies on Tire Tread Block Friction Characteristics Based on a New Test Device," Advances in Meterials Science and Engineering, 2014, 2014, 816204.

[18] Hofstetter, K., Grohs, C., Eberhardsteiner, J. and Mang, H. A., "Sliding Behaviour of Simplified Tire Tread Patterns Investigated by Means of FEM,"
Computers and Structures, 84, 17-18, 2006, 1151-1163.

[19] Costa, H. L. and Hutchings, I. M., "Effects of Die Surface Patterning on Lubrication in Strip Drawing," Journal of Materials Processing Technology, 209, 3, 2009, 1175-1180.

[20] Yuan, S., Huang, W. and Wang, X., "Orientation Effects of Micro-Grooves on Sliding Surfaces," Tribology International, 44, 9, 2011, 1047-1054.

[21] Etsion, I. and Burstein, L., "A Model for Mechanical Seals with Regular Microsurface Structure," Tribology Transactions, 39, 3, 1996, 677-683.

[22] Arghir, M., Roucou, N., Helene, M. and Frene, J., "Theoretical Analysis of the Incompressible Laminar Flow in a Macro-Roughness Cell," Journal of Tribology, 125, 2, 2003, 309-318.

[23] Fowell, M., Olver, A. V., Gosman, A. D., Spikes, H. A. and Pegg, I., "Entrainment and Inlet Suction: Two Mechanisms of Hydrodynamic Lubrication in Textured Bearings," Journal of Tribology, 129, 2, 2006, 336-347.

[24] Nanbu, T., Ren, N., Yasuda, Y., Zhu, D. and Wang, Q. J., "Micro-Textures in Concentrated ConformalContact Lubrication: Effects of Texture Bottom Shape and Surface Relative Motion," Tribology Letters, 29, 3, 2008, 241-252.

[25] Dowson, D., "Elastohydrodynamic and MicroElastohydrodynamic Lubrication," Wear, 190, 2, 1995, 125-138. 